\title{
Closure phase studies toward direct detection of light from hot Jupiters
}

\author{
Ming Zhao ${ }^{1}$, John D. Monnier ${ }^{1}$, Theo ten Brummelaar ${ }^{2}$, Ettore \\ Pedretti ${ }^{3}$, Nathalie Thureau ${ }^{4}$ \\ ${ }^{1}$ Department of Astronomy, University of Michigan, \\ Ann Arbor, MI 48109, USA \\ email: mingzhao@umich.edu \\ ${ }^{2}$ The CHARA Array, Georgia State University \\ ${ }^{3}$ University of St. Andrews, Scotland, UK \\ ${ }^{4}$ University of Cambridge, UK
}

\begin{abstract}
Precision closure phase measurements obtained with ground-based long baseline optical interferometers is a promising way to directly detect light from nearby hot Jupiters. Here we present our closure phase simulations for the CHARA array for several bright hot Jupiters, $v$ And $\mathrm{b}, 51 \mathrm{Peg} \mathrm{b}$, and $\tau$ Boo $\mathrm{b}$. The maximum possible closure phase signals from these hot Jupiters are very small, for example, only $\sim 0.17$ degrees for $v$ And $\mathrm{b}$, requiring very high precision and stable closure phase measurements. We present preliminary results of a closure phase study on test object $\beta$ Tau and hot Jupiter system $v$ And, both obtained with the MIRC instrument at the CHARA array. We demonstrate that directly detecting the light from hot Jupiters is feasible using high precision closure phase measurements obtained by CHARA-MIRC along with its sub-milli-arcsecond resolution, although challenges remain.
\end{abstract}

Keywords. techniques: interferometry, techniques: high angular resolution, exoplanets: hot Jupiters: $v$ And b, 51 Peg b, $\tau$ Boo b

\section{Introduction}

Detecting the light from extrasolar planets is the most direct way to characterize and understand their structures and atmospheres. It is, however, a very challenging task because of the planets' close angular distances and extremely high brightness contrast to their host stars. Among the more than 200 detected extrasolar planets so far, a large population of them are "hot Jupiters", i.e., close-in $(<0.1 \mathrm{AU})$ extrasolar giant planets with high temperatures $\left(T_{\text {eff }}>1000 K\right)$ and large masses $\left(\sim 1 M_{j}\right)$. These "hot Jupiters" are best suited for direct detection and characterization because of their relatively high brightness ratios to their host stars at convenient bands (e.g., J, H, K) and also because of their well-studied atmospheric models. Theoretical models have predicted many features in the atmospheres of hot Jupiters, e.g., molecular bands, dusty clouds, day/night flux variation, etc. (e.g., Burrows et al. 1997; Barman et al. 2005). These features, if confirmed by observations, can provide valuable information on the physical structure, chemical composition and energy distribution of the atmospheres of hot Jupiters (e.g., Barman et al. 2005). To date, four "hot Jupiters" have been directly detected by the Spitzer Space Telescope: HD 209458b, TrES-1, HD 189733b, and most recently, $v$ And b (Deming et al. 2005, 2006; Charbonneau et al. 2005; Harrington et al. 2006). These measurements provided strong constraints to the current planetary atmospheric models and have opened a new era of direct study of these extrasolar planets. However, the number of current measurements is still too few to break model degeneracies and provide 
Table 1. Hot Jupiter candidates for CHARA-MIRC

\begin{tabular}{lllllllllll}
\hline $\begin{array}{l}\text { Star } \\
\text { Name }\end{array}$ & $\begin{array}{l}\text { Dist. } \\
\text { pc }\end{array}$ & $\begin{array}{l}\mathrm{H} \\
\text { mag }\end{array}$ & $\begin{array}{l}\mathrm{K} \\
\text { mag }\end{array}$ & $\begin{array}{l}\text { Period } \\
\text { day }\end{array}$ & & \multicolumn{2}{l}{$\begin{array}{l}\text { Semimajor axis } \\
\text { AU }\end{array}$} & $\begin{array}{l}\mathrm{T}_{0} \\
\text { (mas) }\end{array}$ & $\begin{array}{l}\mathrm{R}_{*} \\
\text { mas }\end{array}$ \\
\hline$v$ And & 13.5 & 2.957 & 2.859 & 4.6170 & 0.034 & 0.059 & $(4.42)$ & 2450088.64 & 0.569 \\
$\tau$ Boo & 15.6 & 3.546 & 3.507 & 3.3128 & 0.018 & 0.049 & $(3.13)$ & 2451653.968 & 0.45 \\
51 Peg & 15.4 & 4.234 & 3.911 & 4.2310 & 0.01 & 0.051 & $(3.31)$ & 2450203.947 & 0.35 \\
\hline
\end{tabular}

References: Marcy, J., et al. 1997; Drake, S., et al. 1998; Butler, P. et al. 1999; Henry, G., et al. 2000; Burrows, A., et al. 2000

detailed conclusions (see e.g., Burrows et al. 2005), and they only provide information in mid-IR. To better understand the physical and chemical characters of these planets, more direct detections are necessary and are undoubtly the best way to proceed.

Among the methods used to detect light from hot Jupiters, a promising way is to use ground-based long baseline optical/IR interferometers along with precision closure phase and/or differential phase measurements. These two methods, especially closure phase, are well studied and have been applied to modern optical/IR interferometers (see, e.g., Segransan et al. 2000; Lopez et al. 2000; Joergens \& Quirrenbach 2004; Monnier 2007 ). These methods are applied in the near infrared so any detections will be very valuable in addition to the Spitzer results. For instance, planetary $\mathrm{T}_{\text {eff }}$ and atmospheric characters can be more accurately determined by combining detections across near and mid-IR bands. In this proceeding we only give a brief introduction to these methods.

Closure Phase. Interferometers obtain information of distant objects through fringe contrast and phase. The presence of a planet causes a small phase shift in the stellar fringe. However, the phase information is always corrupted because the atmospheric turbulence always induces random and extra optical paths to the light. Nevertheless, these extra phases can be canceled if we sum the phases from 3 telescopes in a closed triangle (i.e., $\Delta \Phi_{A B}+\Delta \Phi_{B C}+\Delta \Phi_{C A}=0$, where $\mathrm{A}, \mathrm{B}$, and $\mathrm{C}$ stand for telescopes, see Monnier 1999 for details). This phase closure, or closure phase, is immune to any phase shifts induced by the atmosphere as well as other systematic errors hence is a good observable (Monnier 2007). Closure phase is very sensitive to asymmetry and can sense the structure of starplanet system as analog of high-contrast binaries, which allows us to extract their full orbital parameters (most importantly, the inclination angle) as well as the planet-to-star flux ratio.

Differential Phase. Hot Jupiters and their host stars' brightness contrast differ at different wavelengths (e.g., Sudarsky et al. 2003, Burrows et al. 2007). The difference in the brightness contrast between two wavelengths can cause a slight shift to the system's photo-center, therefore inducing a phase shift to the observed fringe visibility (e.g., Vasisht et al. 2004). This phase shift, also called differential phase, is also immune to the atmospheric turbulence in that the turbulence changes the phases in the same way for different wavelengths, which therefore can be eliminated by the differential measurement. Differential phase can yield the flux ratio and orbital parameters of hot Jupiter systems and, in addition, it also provides valuable spectral information about the planet atmospheres.

In this proceeding we perform simulation studies using both techniques for several hot Jupiters, and show our test observations. Mostly we concentrate on the closure phase method. 

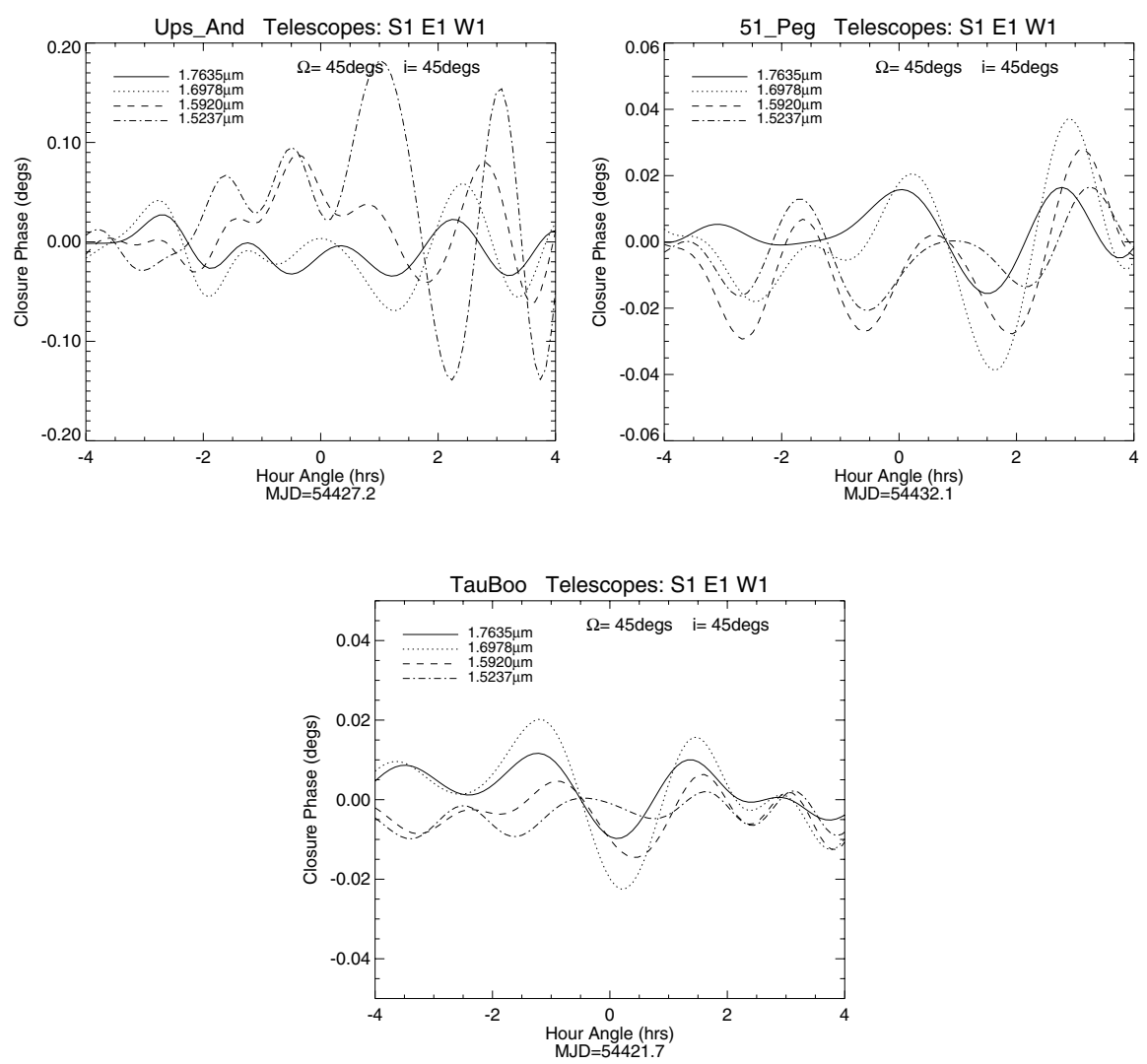

Figure 1. Closure phase simulations for $v$ And b, $51 \mathrm{Peg} \mathrm{b}$ and $\tau$ Boo b. The lines show the results for 4 different wavelength channels (out of 8 in total) of MIRC.

\section{Simulations for nearby hot Jupiters}

Among our list of hot Jupiter candidates chosen for CHARA-MIRC, 3 of them are currently most favorable because of the high brightness of their host stars (see Table 1). Because hot Jupiter-hosting stars and their closest Jupiters are similar to high contrast close binaries, we simulate closure phase signals using binary models (Zhao et al. 2007) for these three systems. The simulated closure phases are obtained using the CHARA interferometer array (ten Brummelaar et al. 2005) along with its MIRC instrument (Monnier et al. 2004) at $\mathrm{H}$ band. The orbital properties of these systems are well studied and are listed in Tab. 1. The diameter of the star $v$ And is newly measured to be $1.138 \pm 0.058$ mas using the FLOUR instrument (Mérand et al. 2006) at CHARA (Mérand 2007, private communication). The infrared flux ratios of the planets are adopted from the models of Sudarsky et al.(2003). The inclination $i$ and the position angle of the ascending node $\Omega$ are unknown for these systems. We assume $45^{\circ}$ inclination and $\Omega=45^{\circ}$ for our first set of models. Figure 1. shows the resulting closure phase simulations for the longest telescope triangle, S1-E1-W1. For $v$ And b (the first panel), we see large variations between channels and the signal level is higher than the other two candidates because its host star is resolved by CHARA, which lowers the fringe visibility of the star and boosts the closure phase. The closure phase of the shortest wavelength channel peaks at $\sim 0.17^{\circ}$ for $v$ And $\mathrm{b}$ and the difference between the first and the last channel is even bigger. For $51 \mathrm{Peg} \mathrm{b}$, 

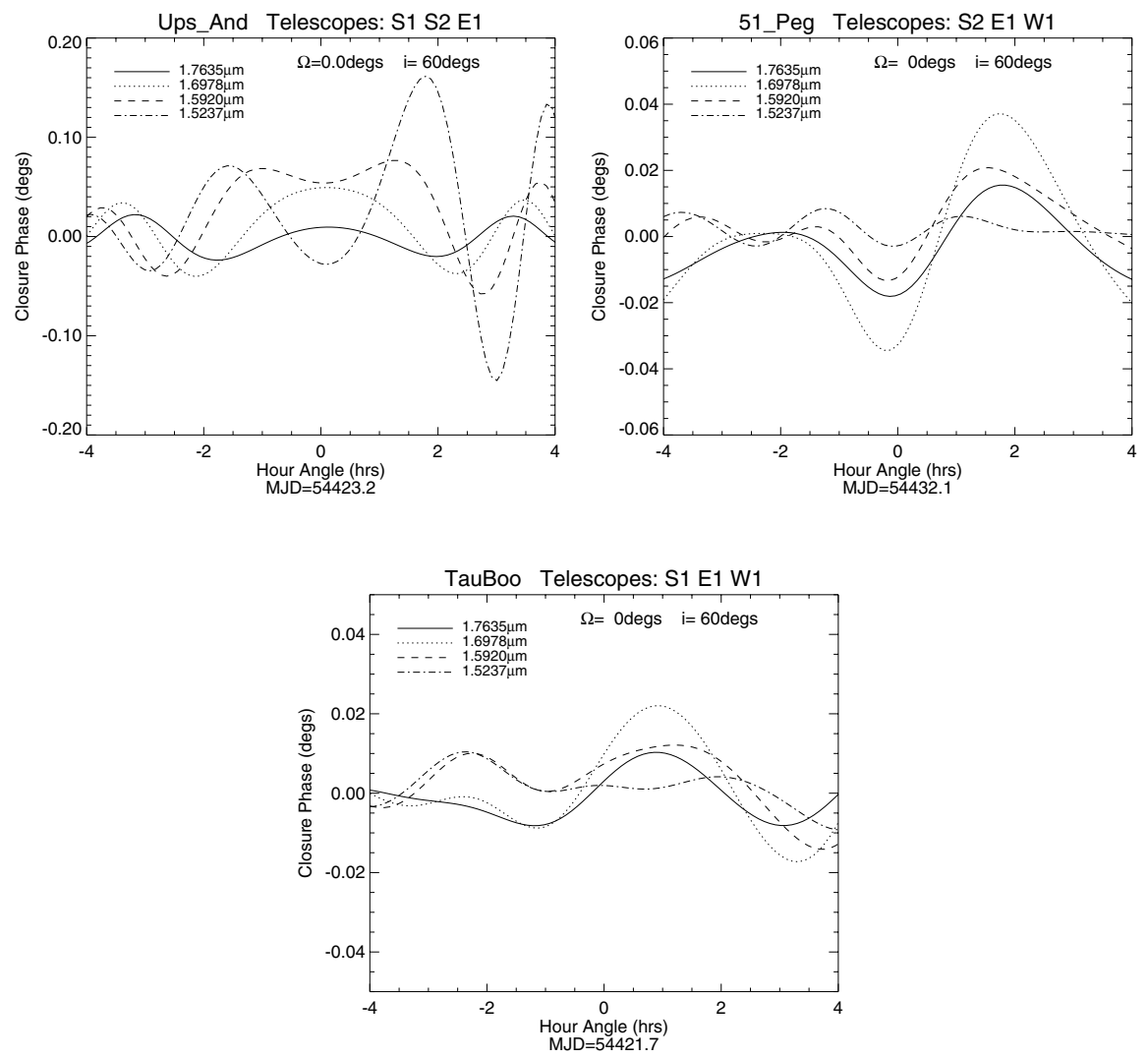

Figure 2. Closure phase simulations for $v$ And b, $51 \mathrm{Peg} \mathrm{b}$ and $\tau$ Boo b, similar to Fig. 1. but for different $i \& \Omega$, i.e., $i=60^{\circ}$ and $\Omega=0^{\circ}$

as shown in the second panel, closure phase peaks at $\sim 0.04$ degs. Because the star 51 Peg is not resolved by CHARA, the closure phase is significantly lower than $v$ And b even though its flux contrast is higher at $\mathrm{H}$ band. For $\tau$ Boo b, although its host star is slightly bigger than $51 \mathrm{Peg}$, its flux ratio is much lower thus the closure phase is weaker and only peaks at $\sim 0.02^{\circ}$.

Although $i$ and $\Omega$ are arbitrarily chosen in Fig. 1, Figure 2 indicates that for a different set of $i$ and $\Omega$ we can always get similar signal levels for the three objects using different telescope triangles and/or at a different date. These plots also suggest that in order to detect such small signals, our precision on closure phases needs to be better than $\sim 0.17^{\circ}$ for $v$ And $\mathrm{b}$ and much better than that for $51 \mathrm{Peg} \mathrm{b}$ and $\tau$ Boo $\mathrm{b}$.

In addition to closure phases, we also simulate differential phases for the three candidates. Specifically, we first simulate complex visibilities for the 8 individual channels, and use the two channels at the edge (i.e., at $1.52 \mu \mathrm{m} \& 1.76 \mu \mathrm{m}$ ) to interpolate the phases for the middle channels to eliminate the OPD drift at different wavelengths due to atmospheric pistons. The differential phase for a certain channel is then calculated by subtracting the interpolated phase. Figure 3 shows the results at $1.66 \mu \mathrm{m}$ (the central channel) using CHARA's longest baseline (S1-E1, 331m). The signals are weaker than closure phases, therefore the required precision for detection is also higher, for instance, need to be better than $0.1^{\circ}$ for $v$ And $\mathrm{b}$. 


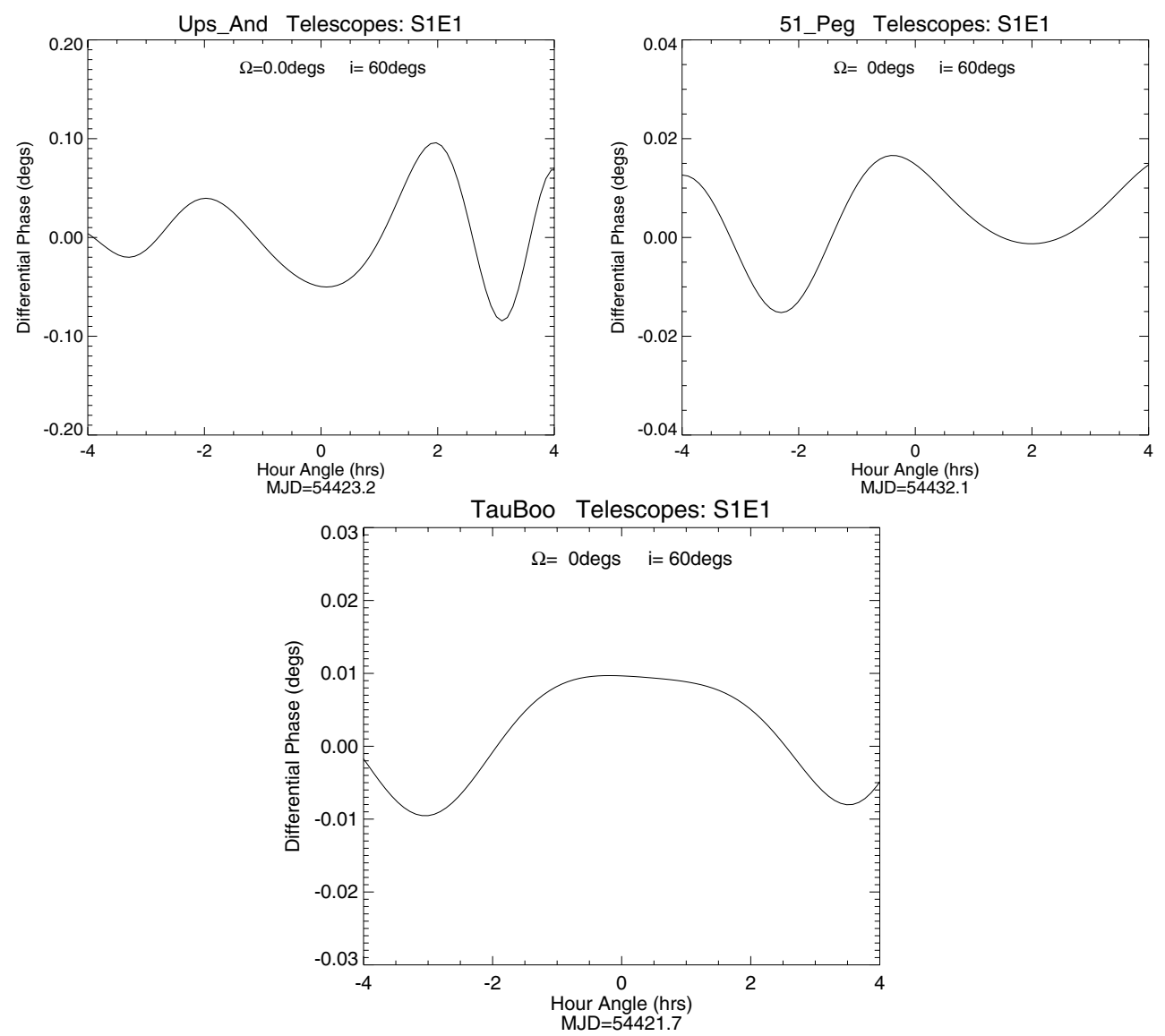

Figure 3. Simulated differential phases for $v$ And b, 51 Peg b and $\tau$ Boo b.

\section{Test Observations}

In order to verify the feasibility of detecting weak signals using CHARA-MIRC as simulated above, we need test observations to study the stability and precision of our measurements. We conducted our first test observation on a bright $\operatorname{star} \beta$ Tau $(\mathrm{H}=1.9)$ on Sep. 15, 2005. The top panel of Fig. 4 shows the closure phase for each data file averaged over all wavelength channels in $\mathrm{H}$ band. The middle panel shows the result of averaging 10 files, and the bottom averages 20 files. The error reduces as $\sqrt{N}$, suggesting the measurement is stable and immune from effects due to changes in the seeing. Encouragingly, the error for $\sim 1.5$ hour integration is $<0.1$ degs, small enough for the required precision, $0.17^{\circ}$, for $v$ And $\mathrm{b}$.

Observations on our first candidate $v$ And was performed in August, 2007. Figure 5 shows the results in 3 panels similar to Fig. 4 . We notice that the error is $0.25^{\circ}$ for only 0.6 hours of integration. Although it is larger than the required precision, the error can in fact reach comparable precision as that of $\beta$ Tau when taking actual integration time and brightness of the star into account. We also notice that the intrinsic errors of the data points are much lower than the scatter and the measurements drift a little with time, which may relate to unknown calibration issues. 


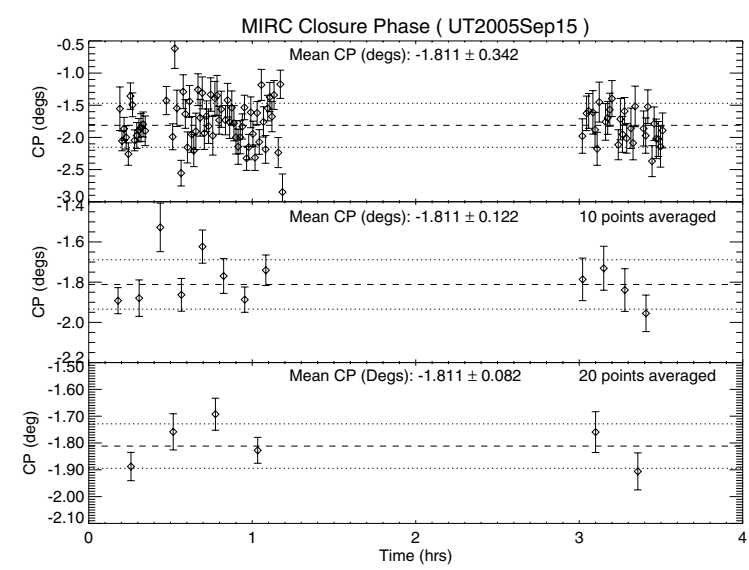

Figure 4. Test observation on bright star $\beta$ Tau. Each point in the top panel corresponds to 45 seconds of integration. The middle panel shows the average of 10 points and the bottom shows the average of 20 points.

\section{Conclusions}

Our simulations show that in order to detect the signal from a hot Jupiter like $v$ And $\mathrm{b}$, the errors of the closure phase and differential phase need to be below $0.1 \mathrm{degs}$ and much lower for other hot Jupiters. Our test observations show that for a bright star with long integration, the precision of our closure phase measurement is enough for the requirement. For a real hot Jupiter, this precision is feasible but requires much longer integration as well as higher sensitivity from the facilities to shorten the integration time. There are indications of some calibration issues in our latest observations. We are investigating these issues to improve the calibration and precision. In the near future, a fringe tracker, CHAMP (Berger et al. 2006), will be commissioned to increase the sensitivity of MIRC, and some optics of the array will be improved with better coating and focusing. With the improvements on both precision and sensitivity, the goal of detecting light from hot Jupiters will be feasible for CHARA-MIRC.

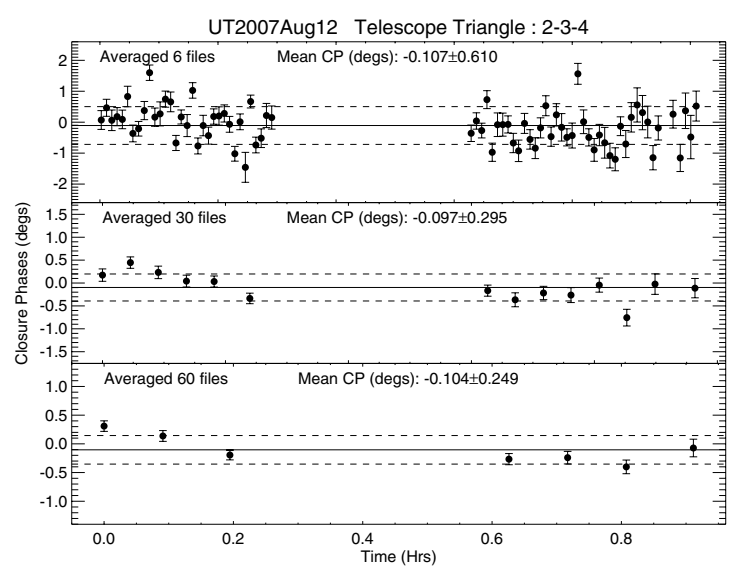

Figure 5. Test observation on hot Jupiter system $v$ And b. Each point in the top panel is an average of 6 files, which corresponds to 32 seconds of integration. Points in the middle panel are averages of 30 files and in the bottom are average of 60 files. 


\section{Acknowledgements}

MZ thanks the support from the Michelson Fellowship. We also thank the support from a NASA-TPF Foundation research grant.

\section{References}

Barman, T. S., Hauschildt, P. H., \& Allard, F. 2005, ApJ, 632, 1132

Berger, D. H., Monnier, J. D., Millan-Gabet, R., ten Brummelaar, T. A., Muirhead, P., Pedretti, E., \& Thureau, N. 2006, in Proceedings of the SPIE, Volume 6268, pp. 62683K (2006).

Burrows, A., Marley, M., Hubbard, W. B., Lunine, J. I., Guillot, T., Saumon, D., Freedman, R., Sudarsky, D., \& Sharp, C. 1997, ApJ, 491, 856

Burrows, A., Hubeny, I., \& Sudarsky, D. 2005, ApJL, 625, L135

Burrows, A., Guillot, T., Hubbard, W. B., Marley, M. S., Saumon, D., Lunine, J. I., \& Sudarsky, D. $2000, A p J L, 534, \mathrm{~L} 97$

Burrows, A., Hubeny, I., Budaj, J., Knutson, H. A., \& Charbonneau, D. 2007, ApJL, 668, L171

Butler, R. P., Marcy, G. W., Fischer, D. A., Brown, T. M., Contos, A. R., Korzennik, S. G., Nisenson, P., \& Noyes, R. W. 1999, ApJ, 526, 916

Charbonneau, D., Allen, L. E., Megeath, S. T., Torres, G., Alonso, R., Brown, T. M., Gilliland, R. L., Latham, D. W., Mandushev, G., O’Donovan, F. T., \& Sozzetti, A. 2005, ApJ, 626, 523

Deming, D., Harrington, J., Seager, S., \& Richardson, L. J. 2006, ApJ, 644, 560

Deming, D., Seager, S., Richardson, L. J., \& Harrington, J. 2005, Nature, 434, 740

Drake, S. A., Pravdo, S. H., Angelini, L., \& Stern, R. A. 1998, AJ, 115, 2122

Harrington, J., Hansen, B. M., Luszcz, S. H., Seager, S., Deming, D., Menou, K., Cho, J. Y.-K., \& Richardson, L. J. 2006, Science, 314, 623

Henry, G. W., Baliunas, S. L., Donahue, R. A., Fekel, F. C., \& Soon, W. 2000, ApJ, 531, 415

Lopez, B., Petrov, R. G., \& Vannier, M. 2000, Proceedings of SPIE, 4006, 407

Marcy, G. W., Butler, R. P., Williams, E., Bildsten, L., Graham, J. R., Ghez, A. M., \& Jernigan, J. G. 1997, ApJ, 481, 926

Mérand, A., Coudé du Foresto, V., Kellerer, A., ten Brummelaar, T., Reess, J.-M., \& Ziegler, D. 2006, in Proceedings of the SPIE, Volume 6268, pp. 62681F (2006).

Monnier, J. D. 1999, in Principles of Long Baseline Interferometry, Edited by Peter R. Lawson, 203-226

Monnier, J. D. 2007, New Astronomy Review, 51, 604

Monnier, J. D., Berger, J.-P., Millan-Gabet, R., \& Ten Brummelaar, T. A. 2004, in Proceedings of SPIE Volume 5491., 2004., p.1370, ed. W. A. Traub, 1370-+

Joergens, V., \& Quirrenbach, A. 2004, Proceedings of SPIE, 5491, 551

Segransan, D., Beuzit, J.-L., Delfosse, X., Forveille, T., Mayor, M., Perrier-Bellet, C., \& Allard, F. 2000, Proceedings of SPIE, 4006, 269

Sudarsky, D., Burrows, A., \& Hubeny, I. 2003, ApJ, 588, 1121

ten Brummelaar, T. A., McAlister, H. A., Ridgway, S. T., Bagnuolo, Jr., W. G., Turner, N. H., Sturmann, L., Sturmann, J., Berger, D. H., Ogden, C. E., Cadman, R., Hartkopf, W. I., Hopper, C. H., \& Shure, M. A. 2005, ApJ, 628, 453

Vasisht, G. \& Colavita, M. M. 2004, in Proceedings of SPIE, Volume 5491., 2004., p.567, ed. W. A. Traub, 567-+

Zhao, M., Monnier, J. D., Torres, G., Boden, A. F., Claret, A., Millan-Gabet, R., Pedretti, E., Berger, J.-P., Traub, W. A., Schloerb, F. P., Carleton, N. P., Kern, P., Lacasse, M. G., Malbet, F., Perraut, K. 2007, ApJ, 659, 626 